\title{
Mobility and QoS-Aware Service Management for Cellular Networks
}

\author{
Omneya Issa \\ Communications Research Centre, Industry Canada \\ Canada
}

\section{Introduction}

As the technologies have evolved in cellular systems from $1 \mathrm{G}$ to $4 \mathrm{G}$, the $4 \mathrm{G}$ system will contain all the standards that earlier generations have implemented. It is expected to provide a comprehensive packet-based solution where multimedia applications and services can be delivered to the subscriber on an anytime, anywhere basis with a satisfactory enough data rate and advanced features, such as, quality of service (QoS), low latency, high mobility, etc. Nevertheless, the 4G cellular system remains a wireless mobile environment, where resources are not given and their availability is prone to dynamic changes. Hence, the basis for QoS provisioning is to control the admission of new and handoff subscriber services in such a way to avoid future detriment perturbation of already connected ones. This task becomes a real challenge when service providers try to raise their profit, by maximizing the number of connected subscribers, while meeting their customer QoS requirements.

The problem can be summarized in that the cellular network should meet the service requirements of connected users using its underlying resources and features. These resources must be managed in order to fulfill the QoS requirements of service connections while maximizing the number of admitted subscribers. Furthermore, the solution(s) must account for the environmental and mobility issues that influence the quality of RF channels, such as, fading and interference. This is the role of service management in cellular networks.

In this chapter, we address service admission control and adaptation, which are the key techniques of service management in mobile cellular networks characterized by restricted resources and bandwidth fluctuation.

Several research efforts have been done for access control on wireless networks. The authors of (Kelif \& Coupechoux, 2009) developped an analytical study of mobility in cellular networks and its impact on quality of service and outage probability. In (Kumar \& Nanda, 1999), the authors have proposed a burst-mode packet access scheme in which high data rates are assigned to mobiles for short burst durations, based on load and interference measurements. It covers burst-mode only assuming that mobiles have only right to one service.

The authors of (Comaniciu et al., 2000) have proposed an admission control for an integrated voice/www sessions CDMA system based on average load measurements. It assumes that all data users have the same bit error rate (BER) requirements. A single cell environment is modeled and no interference is considered. In (Kwon et al., 2003), authors have presented a QoS provisioning framework where a distributed admission control algorithm guarantees the upper bound of a redefined QoS parameter called cell overload probability. Only a single 
class has been investigated; however, interference and fading are not taken into consideration. Also, the authors of (Kastro et al., 2010) proposed a model combining the information about the customer demographics and usage behavior together with call information, yielding to a customer-oriented resource management strategy for cellular networks to be applied during call initiation, handoff and allocation of mobile base stations. Although the model addressed well customer satisfaction within the studied cell, it did not consider interference to other cells.

The authors of (Aissa et al., 2004) proposed a way of predicting resource utilization increase, which is the total received/transmitted power, that would result when accepting an incoming call. Their admission control involves comparing the approximate predicted power with a threshold; this threshold is obtained by determining (offline) the permissible loading in a cell in a static scenario. However, the interference of other cells is not considered in the static scenario and no service adaptation is studied. In (Nasser \& Hassanein, 2004; 2006), despite the fact that the authors have proposed a prioritized call admission control scheme and bandwidth adaptation algorithm for multimedia calls in cellular networks, their framework only supported a single class and only bandwidth is considered in adaptation, which is not tolerated by some multimedia services, such as, voice calls. They did not consider neighbor cell interference as well.

Other research efforts analyzed the soft handoff failure due to insufficient system capacity as done in this chapter. As an example, IS-95 and cdma2000 are compared with respect to the soft handoff performance in terms of outage, new call and handoff call blocking in (Homnan et al., 2000). In (Him \& Koo, 2005), the call attempts of new and handoff voice/data calls are blocked if there is no channel available, and a soft handoff blocking probability is derived as well.

In what concerns the admission policies of handoff calls with respect to new calls, some schemes, such as the ones proposed in (Cheng \& Zhuang, 2002; Kulavaratharasah \& Aghvami, 1999), deploy a guard channel to reserve a fixed percentage of the BS's capacity for handoff users. Other schemes, called nonprioritized schemes in (Chang \& Chen, 2006; Das et al., 2000), handle handoff calls exactly the same way they do with the new calls. Although these approaches are not specially designed, they can be adapted to 3G+ networks as it was briefly represented in (Issa \& Gregoire, 2006) and will be discussed in this chapter.

The above survey has compared state-of-the art admission control proposals, highlighting the main factors of decision making, advantages and weaknesses of different approaches. This leads to pointing out that important challenges pertaining to the wireless environment are yet to be addressed. Therefore, this chapter proposes a strategy that accounts for most of these challenges, such as, cell loading, inter-cell and intra-cell interference, soft handoff as well as QoS requirements in making admission decisions. The strategy also considers the fact that, nowadays, mobile devices are not just restricted to cellular phones; instead, they became small workstations that allow for several simultaneous services per user connection. Factors such as service tolerance for degradation and QoS parameters allowed to be degraded are also exploited. The chapter is organized as follows: in sections 2 and 3 we describe the design details of our approach followed by the design evaluation in section 4 , then we summarize the benefits of the proposal and present future work in section 5 .

\section{Admission control}

Our scheme of service admission on either forward or reverse links is done by measuring the total received or transmitted power at the base station and calculating the available 
capacity according to QoS constraints, interference measurements and fading information gathered from Mobile stations (MSs) in the neighbouring cells. The advantage of building our admission control scheme on power constraints is that it incorporates both bandwidth and BER, represented in target Signal to interference ratio (SIR), since both bandwidth and SIR affect the required channel power and are important in guaranteeing QoS especially in a wireless mobile environment. In addition to power constraints, our admission control follows a policy-based criterion by giving the handoff services priority over the new service connections. In fact, soft handoff attempts should be considered differently from new call attempts because the rejection of handoff attempts from other cells could cause call dropping.

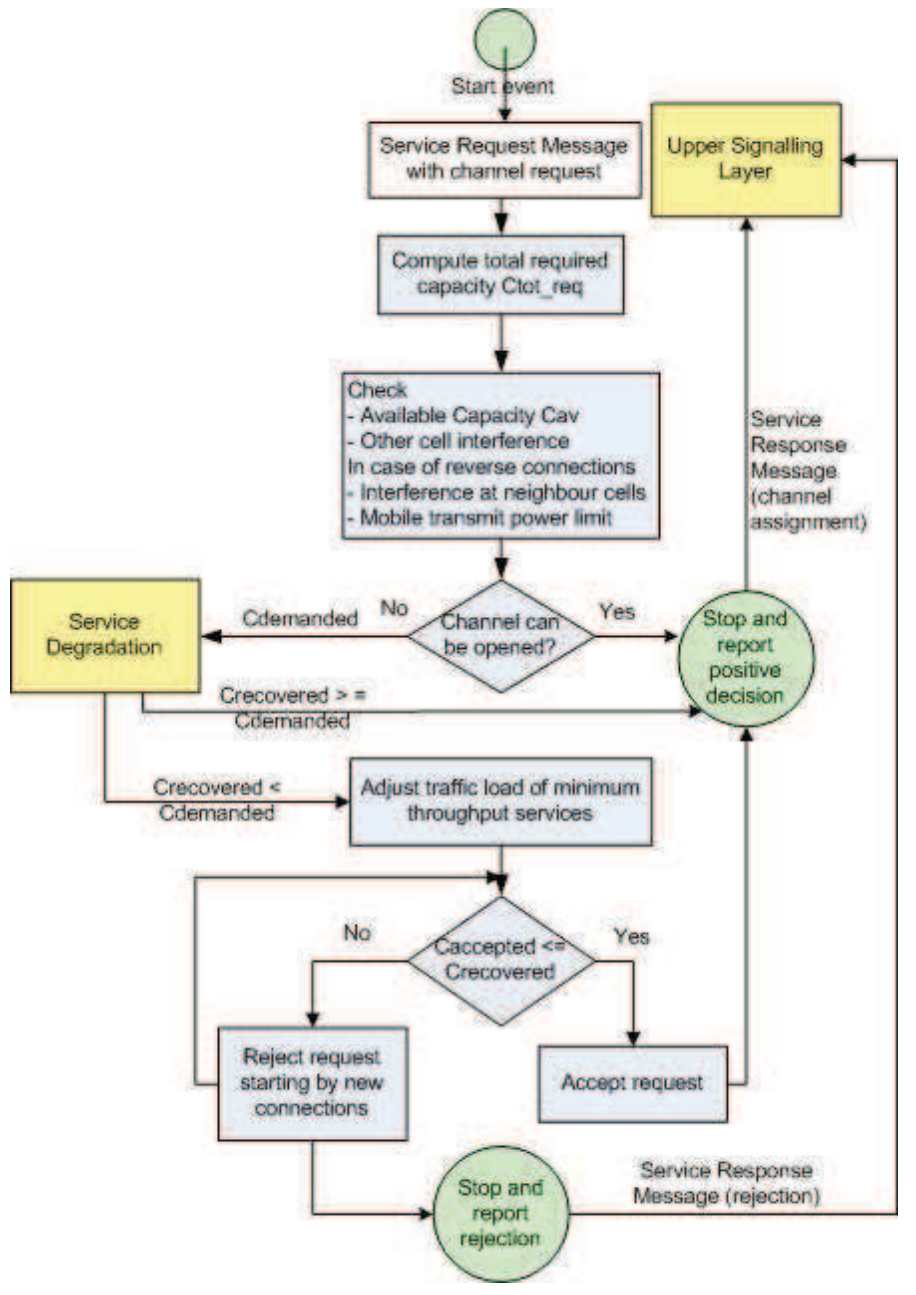

Fig. 1. Admission control scheme

Fig. 1 shows the admission control process. When a new service is required, the base station (BS) admission control (AC) module calculates the required capacity in terms of channel 
power needed, then checks the available capacity taking into account the current service load, the mobile transmit power, the interference of other cells and the interference to neighbour cells. If the available capacity can not cover the initial requirements of the incoming services, the admission control scheme appeals to a degradation procedure for connected services. However, if the degradation process can not recover the needed capacity, the admission control module adjusts the requirements of the incoming services (only services requiring minimum throughput). However, when such adjustment is not enough, it starts rejecting new service requests. Service degradation is discussed in section 3.

We start by describing the verification of load and interference done at beginning of the admission control procedure before appealing to service degradation. The basic idea in resource estimation is actually the same for both uplink (reverse) and downlink (forward).

\subsection{Uplink}

Assuming one BS per cell, this capacity validation procedure is done as follows on the uplink:

$$
P t_{K}=\sum_{j} P_{j, K}+\text { Other_Cell_Interference }+ \text { No, }
$$

where $P t_{K}$ is the total received power by the BS in cell $\mathrm{K}, P_{j, K}$ is the received power at cell $\mathrm{K}$ from $\mathrm{MS}_{j}$ and No is the background noise. As in (Kumar \& Nanda, 1999), $P_{j, K}$ can be written as a function of SIR, i.e. the received ratio of signal bit energy to noise power spectral density $(E b / \mathrm{No})_{j, \mathrm{~K}}$ for $\mathrm{MS}_{j}$ in cell $\mathrm{K}$ divided by its processing gain $\mathrm{G}_{j}$,

$$
P_{j, K}=\frac{1}{G_{j}} P t_{K}\left(\frac{E b}{N o}\right)_{j, K}, \quad G_{j}=\frac{W}{R_{j}},
$$

$W$ is the spreading bandwidth and $R_{j}$ represents the transmission rate of $\mathrm{MS}_{j}$. Including SIR in capacity measurements is very important since $3 \mathrm{G}+$ network is interference limited.

\subsubsection{Interference calculation}

The other interference in cell $\mathrm{K}$ caused by neighbouring cells can be presented in an average sense as a fraction of the in-cell load (Gilhousen et al., 1991), on condition that the load is uniform across all cells. We relaxed this condition to the case where the load in different cells is different, but the average load over all cells is kept fixed to some value by the base station controller (BSC). So (1) can be rewritten as

$$
\begin{aligned}
P t_{K} & =\left(1+\eta_{K}\right) \cdot \sum_{j} P_{j, K}+N o \\
& =P t_{K} \cdot\left(\sum_{j} \frac{1}{G_{j}}\left(\frac{E b}{N o}\right)_{j, K}\right) \cdot\left(1+\eta_{K}\right)+N o,
\end{aligned}
$$

where the other cell interference factor $\eta_{K}$ is defined as in (Kim et al., 2003)

$$
\eta_{K}=\sum_{i \neq K}\left(\frac{1}{M_{i}} \sum_{x=1}^{M_{i}} \eta(x)\right),
$$


$M_{i}$ is the number of MSs in cell $\mathrm{i}$ and $\eta(x)$ is calculated as

$$
\eta(x)=\frac{\rho_{K}(i, x) L_{K}(i, x)}{\rho_{i}(i, x) L_{i}(i, x)}
$$

where $\rho_{K}(i, x)$ is the fast (Rayleigh) fading and is given by

$$
\rho_{K}(i, x)=\sum_{p=1}^{P} g_{K, x(i), p^{\prime}}^{2}
$$

with $g_{K, x(i), p}^{2}$ is the $p^{\text {th }}$ path gain between $\mathrm{BS}_{K}$ and $\mathrm{MS}_{x}$ in cell $\mathrm{i}$, and $L_{K}(i, x)$ presents slow fading which is modeled as

$$
L_{K}(i, x)=r_{K, x(i)}^{-\delta} \cdot 10^{\xi_{K, x(i)}} / 10,
$$

where the signal between $\mathrm{BS}_{K}$ and $\mathrm{MS}_{x}$ in cell $\mathrm{i}$ experiences an attenuation by the $\delta$ th power of the distance $r_{K, x(i)}$ between $\mathrm{BS}_{K}$ and $\mathrm{MS}_{x}$ and log-normal shadowing ( $\xi$ is a zero-normal variant with standard variation $\sigma$ ).

The uplink capacity is directly affected by the noise rise generated by users in the uplink. The noise rise $N_{r}$ is the increase in noise compared to the noise floor of the cell; thus:

$$
N_{r}=\frac{P t_{K}}{N o}
$$

The concept of noise rise means that infinite noise rise must be considered when the load is $100 \%$ (e.g. the pole capacity). Hence, $N_{r}$ can be written as a function the cell uplink load $C_{U}$; when $C_{U}$ is close to unity, the noise rise approaches infinity as shown in (9):

$$
N_{r}=\frac{1}{1-C_{U}}
$$

From (8), the $C_{U}$ can be written in function of the total received power as follows:

$$
C_{U}=\frac{P t_{K}-N o}{P t_{K}}
$$

Using (3),

Recall that from (2),

$$
C_{U}=\left(1+\eta_{K}\right) \cdot \frac{\sum_{j} P_{j, K}}{P t_{K}},
$$

So (11) becomes:

$$
\frac{P_{j, K}}{P t_{K}}=\frac{R_{j}}{W}\left(\frac{E b}{N o}\right)_{j, K},
$$

$$
C_{U}=\left(1+\eta_{K}\right) \cdot \sum_{j} \frac{R_{j}}{W}\left(\frac{E b}{N o}\right)_{j, K}{ }^{\prime}
$$

(13) assumes only one channel per MS. To extend it to the case, as for wideband CDMA, where an MS can have several channels with different target SIR's, data rates and activity factors, (13) can be written as

$$
C_{U}=\left(\frac{1}{W} \sum_{j} \sum_{n=1}^{N_{j}} R_{n, j, K}\left(\frac{E b}{N o}\right)_{n, j, K} a_{n, j, K}\right) \cdot\left(1+\eta_{K}\right),
$$


where $N_{j}$ is the number of channels of $\mathrm{MS}_{j}$, and $(E b / \mathrm{No})_{n, j, K}$ and $a_{n, j, K}$ are the received $\mathrm{Eb} / \mathrm{No}$ and the activity factor for service of channel $\mathrm{n}$ of $\mathrm{MS}_{j}$ in cell $\mathrm{K}$ respectively.

So the admission condition for accepting the uplink connection(s) of $\mathrm{MS}_{i}$ in cell $\mathrm{K}$ is

$$
\begin{array}{r}
\frac{\left(1+\eta_{K}\right)}{W}\left(\sum_{j \neq i} \sum_{n=1}^{N_{j}} R_{n, j, K}\left(\frac{E b}{N o}\right)_{n, j, K} a_{n, j, K}\right) \\
+\frac{\left(1+\eta_{K}\right)}{W}\left(\sum_{n=1}^{N_{i}} R_{n, i, K}\left(\frac{E b}{N o}\right)_{n, i, K} a_{n, i, K}\right) \leq T h_{U},
\end{array}
$$

where $N_{i}$ is the number of channels of $\mathrm{MS}_{i},(E b / \mathrm{No})_{n, i, K}$ is the required $\mathrm{Eb} / \mathrm{No}$ (SIR) for channel $\mathrm{n}$ of $\mathrm{MS}_{i}$ in cell $\mathrm{K}$ and $\eta_{K}$ is computed by (4-7). Theoretically, Th $h_{U}$ is equal to 1 , which is the pole capacity. However, an operator restricts the uplink load to a certain noise rise, hence, practically $T h_{U}$ is kept below unity.

\subsection{Downlink}

The downlink cell capacity follows the same logic as the uplink. The total base station transmit power, Ptx_tot $K_{K}$, is estimated. It is the sum of individual transmit powers required for downlink connections of MSs in a cell as shown below.

$$
\text { Ptx_tot }{ }_{K}=\sum_{j} P t x_{j, K}
$$

where $P t x_{j, K}$ is the downlink power required for MS $j$ in cell $K$, assuming only one service per mobile. Ptx $_{j, K}$ is given by (Sipila et al., 2000):

$$
\operatorname{Ptx}_{j, K}=\frac{R_{j}}{W}\left(\frac{E b}{N o}\right)_{j, K} \cdot\left((1-f) \text { Ptx_tot }_{K}+\eta_{j} \cdot \text { Ptx_tot }_{K}+N o \cdot L_{K, j}\right),
$$

where $L_{K, j}$ is the path loss from base station $K$ to $\mathrm{MS}_{j}$ and $f$ is the orthogonality factor modeling the intracell interference from non-orthogonal codes of other MSs, using $f=1$ for fully orthogonal codes and 0 as not orthogonal. Note that the orthogonality factor $f$ depends on the codes used for users inside a cell, and even if these codes are perfectly orthogonal, there is always some degree of interference between the signals of mobiles of the same cell due to multi-path. Delayed copies received from a multipath fading are not orthogonal any more and cause multipath fading interference, which is modeled as a factor of the total base station transmit power. For simplicity, we do not consider the orthogonality factor of each code; we take $f$ as the average orthogonality factor in the cell. $\eta_{j}$ is the other-cell-to-own-cell received power ratio (inter-cell interference) for $\mathrm{MS}_{j}$ modeled as a factor of the total downlink power and calculated as follows:

$$
\eta_{j}=\sum_{i \neq K} \frac{\rho_{K, j} L_{K, j}}{\rho_{i, j} L_{i, j}}
$$

where $\rho_{K, j}$ and $L_{K, j}$ are the fast and slow (path loss) fading from the serving base station $K$ to $\mathrm{MS}_{j}$ and $\rho_{i, j}$ and $L_{i, j}$ are the fast and slow (path loss) fading from another base station $i$ to $\mathrm{MS}_{j}$ respectively.

The other-to-own-cell interference on the downlink depends on mobile location and, therefore, is different for each mobile. However, the estimation of the downlink transmission power should be on average basis and not on the maximum transmission power at the cell 
edge. The average transmission power per mobile is determined by considering the user at an average location in the cell. Thus, we may let $\eta$ be the average other-to-own cell interference seen by the mobile as in (Sipila et al., 2000):

$$
\eta=\frac{1}{J} \sum_{j} \eta_{j}
$$

where $J$ is the number of MSs served by the base station.

By summing up $P t x_{j, K}$ over the number of MSs, Ptx_tot $t_{K}$ can be derived as in (20). Note that in this estimation the soft handover must be included. thus, $j$ must include the soft handover connections, which are modeled as additional connections in the cell, as well.

$$
\text { Ptx_tot }_{K}=\frac{N o \sum_{j} \frac{R_{j}}{W}\left(\frac{E b}{N o}\right)_{j, K} L_{K, j}}{1-\sum_{j} \frac{R_{j}}{W}\left(\frac{E b}{N o}\right)_{j, K} \cdot((1-f)+\eta)},
$$

Using the same reasoning on the noise rise as for the uplink, we can define the cell downlink load $C_{D}$ as follows (Sipila et al., 2000):

$$
C_{D}=\sum_{j} \frac{R_{j}}{W}\left(\frac{E b}{N o}\right)_{j, K} \cdot((1-f)+\eta),
$$

When $C_{D}$ is close to 1 , the base station transmit power approaches infinity. To extend (21) so that an MS can have several channels with different activity factors, we obtain the admission condition for the downlink connection of MSi by:

$$
\begin{array}{r}
\frac{(1-f+\eta)}{W}\left(\sum_{j \neq i} \sum_{n=1}^{N_{j}} R_{n, j, K}\left(\frac{E b}{N o}\right)_{n, j, K} a_{n, j, K}\right) \\
+\frac{(1-f+\eta)}{W}\left(\sum_{n=1}^{N_{i}} R_{n, i, K}\left(\frac{E b}{N o}\right)_{n, i, K} a_{n, i, K}\right) \leq T h_{D},
\end{array}
$$

Theoretically, $T h_{D}$ is equal to unity, which is called the pole capacity. However, lower $T h_{D}$ values will be tested to limit the noise rise. Note that in case of no orthogonality $(f=0),(22)$ becomes similar to the uplink case.

\subsection{Reverse connections and soft handoff}

In reverse connections, a MS can have more than one leg in soft handoff. So, in addition to the procedure proposed above, to account for soft handoff, the following conditions must be satisfied:

- $\quad j$ in (15) is summed over the set of MSs that have cell $\mathrm{K}$ in their active set.

- (15) must be satisfied for each soft handoff leg of $\mathrm{MS}_{i}$.

- Since adjacent-cell interference is critical in deciding for the admission of reverse connections, it is necessary to evaluate the interference at the non active cells caused by the admission of the reverse connection. So, interference constraints at neighbour cells that are not in the active or candidate set of $\mathrm{MS}_{i}$ should be satisfied. 
Pilot strength information received at $\mathrm{MS}_{i}$ for cells in its neighbour list can indicate to BS the interference levels that will be seen at its neighbour BSs due to transmissions from $\mathrm{MS}_{i}$. The MS reports pilot strength information for cells in its neighbour list in the Paging Channel. So, to avoid producing excessive interference at a neighbour cell NC, we constrain the path loss difference between the strongest active and strongest non-active pilots to a minimum $\Delta$ (Kumar \& Nanda, 1999) such that

$$
\begin{gathered}
P S_{0}-P S_{N C} \geq \Delta, \\
P S_{0}=\max _{K}\left(P S_{i K}\right), \quad K \in A S, \\
P S_{N C}=\max _{K}\left(P S_{i K}\right), \quad K \notin A S \text { and } K \in N S,
\end{gathered}
$$

where $P S_{i K}$ is the pilot strength reported by $\mathrm{MS}_{i}$ from cell $\mathrm{K}, \mathrm{AS}$ is the set of active and candidate pilots and NS is the set of neighbour pilots. $P S_{0}$ is the strength of the strongest active pilot and $P S_{N C}$ is the strength of the strongest non-active pilot.

\subsection{User equipment transmit power}

Another factor that must be taken into account, is the limited transmission power available at the mobile stations. Here, we do not model battery lives, however, we check if the transmit power of the mobile station, required to meet the uplink target $\mathrm{Eb} / \mathrm{No}$, does not exceed the maximum mobile transmit power. As indicated in Fig.1, after the uplink cell load is checked, the required mobile transmit power is verified with respect to its maximum. The required mobile transmit power $\operatorname{Ptx}_{M S_{i, K}}$ of MSi can be computed as follows (Sipila et al., 2000):

$$
\operatorname{Ptx}_{M S_{i, K}}=\frac{N o\left(\sum_{n=1}^{N_{i}} R_{n, i, K}\left(\frac{E b}{N o}\right)_{n, i, K} a_{n, i, K}\right) L_{i, K}}{1-C_{U}},
$$

where $N_{i}$ is the number of uplink channels of $\mathrm{MS}_{i}$. A mobile station, which is not able to transmit with the required amount of power to meet the required Eb/No due to maximum power limitations is not admitted (blocked). Note that incoming service requests can be blocked either because of noise rise limits (equations (15) and (22)) or in case of limited mobile transmit power. It is worth noting that the limitation of total base station power is already considered by applying lower values of the threshold $T h_{D}$ in (22).

\subsection{Recalculation of admitted load}

A new channel can be assigned by sending a Channel Assignment Message with Service Response Message to MS if all the above conditions are met. However, if all the conditions are satisfied except the uplink condition (15) or the downlink one (22), the admission module asks the Degradation module to try to acquire the capacity left to satisfy the required one.

In order to compute the capacity required from the Degradation module, (15) and (22) are interpreted in terms of the available capacity. The available capacities $C_{a v_{U}}$ and $C_{a v_{D}}$ for accepting services on the uplink (U) and downlink (D) respectively can be given by:

$$
C_{a v_{U}}=\frac{W \cdot T h_{U}}{\left(1+\eta_{K}\right)}-\left(\sum_{i} \sum_{n=1}^{N_{i}} R_{U_{n, i, K}}\left(\frac{E b}{N o}\right)_{U_{n, i, K}} a_{U_{n, i, K}}\right),
$$




$$
C_{a v_{D}}=\frac{W \cdot T h_{D}}{((1-f)+\eta)}-\left(\sum_{i} \sum_{n=1}^{N_{i}} R_{D_{n, i, K}}\left(\frac{E b}{N o}\right)_{D_{n, i, K}} a_{D_{n, i, K}}\right),
$$

where $i$ is summed over the set of existing (already connected) MSs.

Since the first term in (25) and (26) does not depend on the service requirements, for simplicity, we define a variable $C$ that accounts for the second term that varies according to services; it will be referred to as 'service capacity'. Thus, the total required service capacity for new/handoff requests, for uplink and downlink, can be written as:

$$
C_{\text {tot_req }_{U / D}}=\left(\sum_{j} \sum_{n=1}^{N_{j}} R_{(U / D)_{n, j, K}}\left(\frac{E b}{N o}\right)_{(U / D)_{n, j, K}} a_{(U / D)_{n, j, K}}\right),
$$

where $j$ is summed over the set of the new/handoff MSs and $N_{j}$ is the number of services on either the uplink or the downlink. If $C_{\text {tot_req }}$ can not be satisfied by the available capacity, the admission control appeals service degradation with the uplink and downlink demanded service capacities, $C_{d_{U}}$ and $C_{d_{D}}$ respectively. The demanded capacity is the difference between the required capacity and the available one:

$$
\begin{gathered}
C_{d}=C_{\text {tot_req }}-C_{a v}, \\
C_{d_{U}}=\left(\sum_{j} \sum_{n=1}^{N_{j}} R_{U_{n, i, K}}\left(\frac{E b}{N o}\right)_{U_{n, i, K}} a_{U_{n, i, K}}\right)-\frac{W \cdot T h_{U}}{\left(1+\eta_{K}\right)}, \\
C_{d_{D}}=\left(\sum_{j} \sum_{n=1}^{N_{j}} R_{D_{n, i, K}}\left(\frac{E b}{N o}\right)_{D_{n, i, K}} a_{D_{n, i, K}}\right)-\frac{W \cdot T h_{D}}{((1-f)+\eta)},
\end{gathered}
$$

where $j$ is summed over the set of existing MSs as well as the new / handoff ones.

If the capacity recovered by service degradation, $C_{\text {recovered }}$ can provide the demanded capacity, the service requests are accepted. However, to account for a worst case scenario where the Degradation module cannot deliver the demanded capacity, the AC module, in order to maximize the number of admitted services, recalculates the required capacity by trying to decrease the accepted load of new/handoff minimum throughput services only, since bounded delay services such as voice and video cannot tolerate such process. However, the accepted load of each minimum throughput service is reduced, if possible, by an equal share of unacquired capacity. In other words, each minimum throughput service will have an equal share of the total now available capacity $\left(C_{a v}+C_{\text {recovered }}-C_{r e q B D}\right)$ with respect to the required capacity of all the new/handoff minimum throughput services. $C_{r e q B D}$ is the capacity required for bounded delay new/handoff services. Note that the service requirements are not decreased below the QoS limits, $C_{\text {min }}$, indicated in its QoS profile. Thus, the accepted capacity of service $n$ is the greater of two values, its minimum required capacity and its share of the total available capacity:

$$
C_{n \_ \text {accepted }}=\max \left(C_{n \_ \text {min }}, C_{n \_ \text {req }} \cdot\left(C_{a v}+C_{\text {recovered }}-C_{\text {reqBD }}\right) / \sum_{j=1}^{N} C_{j \_r e q}\right),
$$

where $C_{n}=R_{n}(E b / N o)_{n} a_{n}$, where $R_{n}$ and $(E b / N o)_{n}$ are the rate and the SIR of the channel needed for service $n$ respectively, and $a_{n}$ is the service activity factor. $N$ is the number of 
new/handoff minimum throughput services. It will be seen in the next section that the load of minimum throughput services is decreased by adjusting their rate $R$.

It can happen, even when reducing the capacity of minimum throughput services, that the accepted capacity exceeds available plus recovered capacities because we do not reduce the service capacity below the limits of its QoS profile. So the last possible course of action is to reject requests, as in Fig.1. We begin by rejecting new service requests since forced termination of handoff services has significant negative effect on the user's perception of network reliability, and, therefore, affect subscribers' expectation.

It is worth noting that the admission control scheme is simple to apply and has a low computation time. The time complexity for calculating conditions (15) and (22) is of order $O((N c+1) M)$ and the one for reducing the accepted load of new/handoff minimum throughput services is of order $O\left(N_{\min T}\right)$ where $N c$ in the number of neighbour cells, $M$ in the average number of MSs per cell and $N_{\min T}$ is the number of new/handoff minimum throughput services. So the time complexity of our admission module is of order $O((N c+$ 1) $\left.M+N_{\min T}\right)$, that is $O(M)$ since $N c$ is limited to a few cells. In fact, the complexity of the proposed approach is considered low with respect to the inclusion of a realistic interference computing. Other complex approaches adopt a global call admission control where the actual interference should be computed between all cells; their computational complexity is of order of $O\left(N^{2}\right)$ where $N$ is the number of cells in the network. On the other hand, more simplistic admission control algorithms simply consider the calls currently active in the intended cell in order to accept or reject a new call. The inter-cell interference is either neglected or sometimes considered as a constant factor. These algorithms usually have the lowest computational complexity of $O(1)$, which is an optimal complexity, however, at the cost of not considering realistic conditions.

\section{Degradation and improvement}

The problem of high new call blocking and handoff dropping can be partially solved by QoS adaptation (degradation) which is much more bearable to users than a forced termination of their services. The improvement module is important for deciding the appropriate distribution of resources freed by terminating and ongoing services. Also, the admission control needs this module in order to make room for admitting new and handoff connections. In (Lee et al., 2000) authors have proposed an adaptive resource allocation mechanism that allocates connection resources for incoming calls utilizing bandwidth degradation and compensation. Also (Kwon et al., 2003) have proposed a bandwidth adaptation algorithm which seeks to minimize a redefined QoS parameter called cell overload probability. Both adaptation frameworks only take mobility into consideration; they do not account for fading and interference. Both approaches consider only the transmission rate as the only parameter to degrade. Moreover, all calls that exceed their average or minimum bandwidth are degraded to their average or minimum bandwidth respectively. This requires a large number of signalling messages.

\subsection{Limiting signalling overhead}

Service degradation/improvement implies changing resources assignment. This can be a time and bandwidth-consuming process, because the number of degraded/ improved services increases the amount of required signalling messages respectively. So we have decided to limit the number of degraded/improved services by selecting only MSs located in a certain zone of the cell. 
In fact, other criteria than MS locations can be used to limit the number of adapted services such as the degraded time of services. But, since our objective is to maximize the number of admitted services while maintaining QoS, we should minimize interference as well. To achieve this, the cell is divided into two zones: zone 1 where the distance of MS from the BS, located in the center of the MS cell, is higher than a value Rsafe, and zone 2 where this distance is lower than Rsafe.

In our design, MSs located in zone 1 can be degraded. Since degradation means reducing resources, i.e. transmission power, so degrading services of MS located near the cell boundary, in zone 1, would decrease interference to adjacent cells. On the other hand, since improvement implies increasing transmission power, services of MS located in zone 2, far from cell boundary, can be improved without causing interference to neighbour cells. The prediction of MS location can be done based on the pilot signal strength from BS to MS on the Paging Channel or on the phase delay of the received signal. We call zone 2 the safe region because services are not degraded if their MS is in this zone.

The proposed policy for degradation and improvement mainly aims to limit interference and, hence, maximize the admission probability. Nevertheless, this also leads to unfairness in distributing radio resources among the mobiles of the same cell. MSs far from the BS are more likely subject to degradation while mobiles near the BS are favored for improvement.

Note that the nomenclature of signalling messages are based on cdma2000 signalling messages specified in (C.S0005-Ev2.0, 2010). This can be easily mapped to signalling messages of WCDMA and LTE.

\subsection{QoS level adjustment}

Service degradation/improvement (D/I) also implies the adjustment of the QoS level. QoS is adjusted by changing the data rate or the target BER according to service tolerance. Both kinds of changes lead to changing the transmission power because modifying the data rate modifies the channel processing gain, and modifying the BER leads to the alteration of the target $\mathrm{Eb} / \mathrm{No}$. We have decided to adjust the QoS of non real-time services (those requiring only a minimum throughput) by changing their data rate according to their profile since they cannot tolerate higher BER degradation. The QoS of real-time services is adjusted by modifying their Eb/No because they can tolerate such change, however, a variation in their data rate implies a delay change which is not tolerable by such services. We have decided not to alter the transmission power of voice services, whether by changing their bit rates or their target $\mathrm{Eb} / \mathrm{No}$, because imposing any degradation of voice traffic would harm its QoS requirements.

Moreover, our design respects the limited tolerance to variation of real-time services. So, in the Degradation module presented in Fig.2, we begin by degrading the minimum throughput services. The real-time services, such as near real-time video (NRTV) services, are not degraded unless there is no minimum throughput service left to be degraded. Also, when MSs finish their call or leave the cell, the Improvement module, shown in Fig.3, shares out the freed capacity $C_{\text {freed }}$ beginning by improving the degraded NRTV services, then, the remaining capacity, $C_{f r}$ left, goes to the degraded minimum throughput services that are still in the safe region. Note that the degraded minimum throughput services get an equal share of the surplus capacity with respect to the required capacity of the degraded minimum throughput services. Thus, as in (30), the new allocated rate to a minimum throughput service $n$ is the lower of two values, its required rate $R_{n_{-}} r e q$ and its share of the left freed capacity added to its 


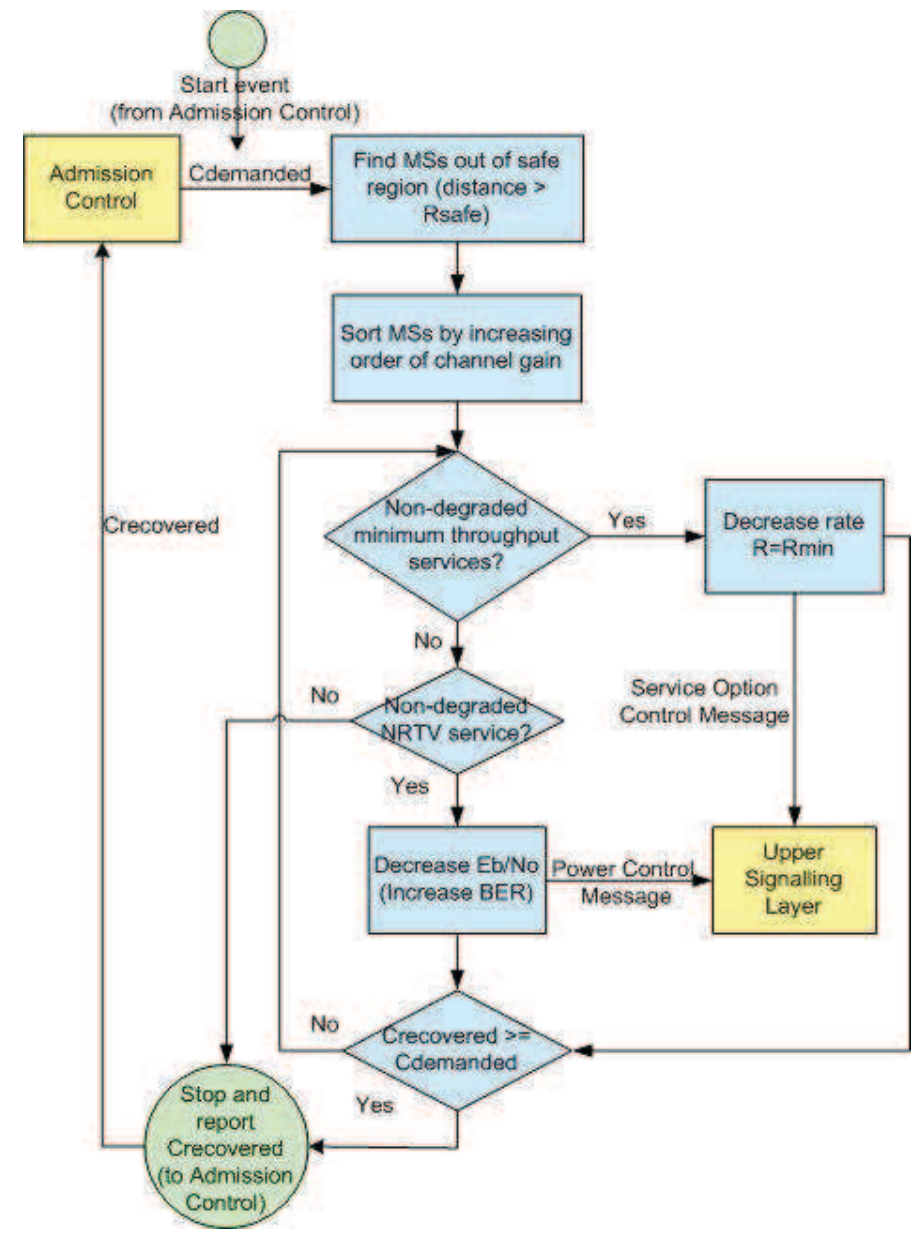

Fig. 2. The Degradation module

actual rate $R_{n \_} a$ :

$$
R_{n}=\min \left(R_{n \_r e q},\left(R_{n \_a}+\left(R_{n \_r e q} \cdot C_{f r \_l e f t}\right) / \sum_{j=1}^{N} C_{j \_r e q}\right)\right),
$$

where $N$ is the number of degraded minimum throughput services in zone 2 .

\subsection{Limiting interference}

In addition to limiting signalling overhead, we have optimized our Degradation module to further minimize the interference to adjacent cells. Services to be degraded are sorted by ascending order of their channel gain, where the channel gain $G_{x, K}$ of channel $\mathrm{x}$ in cell $\mathrm{K}$ is given by

$$
G_{x, K}=\rho_{x}(K, x) L_{x}(K, x),
$$




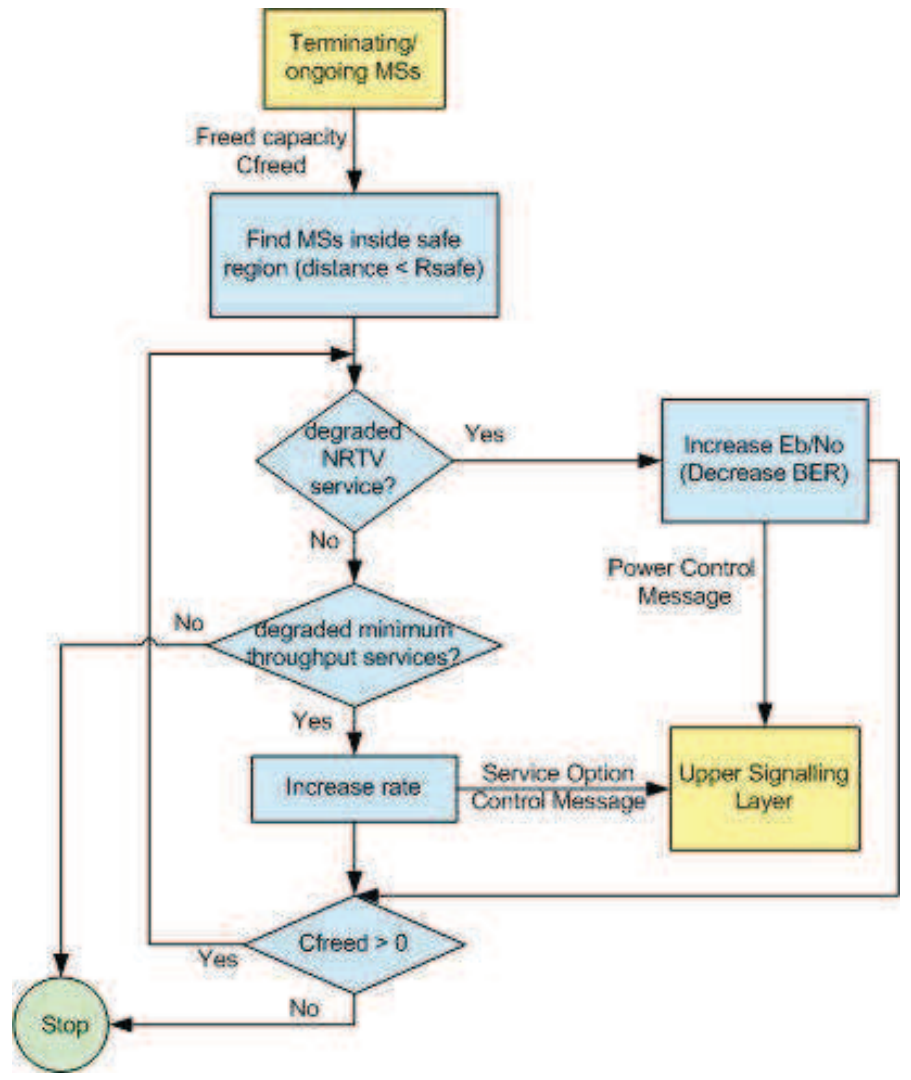

Fig. 3. The Improvement module

where $\rho_{x}$ and $L_{x}$ are calculated by (6) and (7). Since MSs located near cell boundary most likely have lower channel gain, allocating lower transmission power to these MSs would decrease interference to neighbour cells. So, we begin by degrading services having the lowest channel gain, hence, assigning to them lower transmission power and thus, limiting interference to adjacent cells.

When called by the admission control, the Degradation module presented in Fig.2 starts by finding the MSs in zone 1 then sorts them by increasing order of channel gain. It begins by adapting non-degraded minimum throughput services by decreasing their rate. The real-time services are degraded when there is no minimum throughput one left. Thereafter, the $C_{\text {recovered }}$ is reported to the admission control. Reciprocally, as shown in Fig.3, in case of terminating or ongoing MSs, the Improvement module equally distributes the freed capacity first among MSs in zone 2. It starts by the degraded real-time services then the minimum throughput ones according to (30).

The D/I modules have a low computation time. Locating MSs in safe region is of the order $O(N)$ where $N$ in the number of MSs in the cell. Sorting selected MSs by their channel gain has a maximum computation order $O(N \log N)$. So the time complexity of the Improvement module is of order $O(N)$ and the upper bound of time complexity of the degradation module 
is of order $O(N \log N)$. This order of complexity is comparable to the order of any service adaptation approach that deploys some selection criteria among MSs in a single cell. It is lower than the complexity of the approaches that apply global adaptation among all the cells of the network. Their complexity is of the order of $O(M \times N)$ where $M$ is the number of cells and $N$ is the average number of MSs/cell.

\section{Design evaluation}

To test our proposed modules, we have built a simulation model that includes mobility, fading and interference.

Our cellular system consists of 19 hexagonal cells, with a BS in the center of each cell. The reported results are the ones of the central cell; the other 18 cells are source of interference around it. Typically, two rings are sufficient to generate significant interference to the MSs in the central cell.

Two environments are simulated: an indoor environment in an office with soft partitions and an outdoor environment in a shadowed urban area. In order to account for fading as well as mobility, slow and fast fading are simulated in the channel model, since they affect the signal strength of the MS channel measured by BS. Other parameters of fading and mobility simulation are shown in Table 1.

The simulation of soft handoff follows the IS-95 algorithm. At each MS, the active pilots are determined by computing the pilot signal strength received from all BSs. MS is assumed to be in handoff with all BSs having an average pilot signal strength within $6 \mathrm{~dB}$ of the strongest serving site, so $\Delta$ is set to $6 \mathrm{~dB}$ in (23). The parameters of active and candidate set evaluation are: $T_{A d d}=-12 d B, T_{\text {Drop }}=-16 d B$ and $T_{T_{\text {Drop }}}=3 \mathrm{~s}$. In soft handoff, the transmit power is allocated on uplink and downlink according to the strongest base station pilot.

\begin{tabular}{|c|c|c|}
\hline Parameter & $\begin{array}{c}\text { Indoor } \\
\text { (Office) }\end{array}$ & $\begin{array}{c}\text { Outdoor } \\
\text { (Vehicular) }\end{array}$ \\
\hline Time between direction & $1 \mathrm{~min}$ & $1 \mathrm{~min}$ \\
changes T & & \\
Cell radius Rc & $50 \mathrm{~m}$ & $5 \mathrm{~km}$ \\
Movement direction $\alpha$ & $\pi / 4$ & $\pi / 4$ \\
Velocity & $5 \mathrm{~km} / \mathrm{h}$ & $100 \mathrm{~km} / \mathrm{h}$ \\
Velocity standard deviation & $3 \mathrm{~km} / \mathrm{h}$ & $5 \mathrm{~km} / \mathrm{h}$ \\
Path loss exponent $\delta$ & 1.6 & 3.0 \\
Shadowing deviation $\sigma$ & 9.6 & 4.0 \\
Number of paths P & 2 & 2 \\
\hline
\end{tabular}

Table 1. Simulation parameters

Requests are made for service connection establishment at a service arrival rate $\lambda$. Forward and reverse services are uniformly distributed on MSs; each MS can have up to 3 different services. Voice and NRTV are the simulated real-time services with BER of $10^{-3}$ and $10^{-4}$ and target $E b / N o$ of 7 and 8dB respectively (TR45.5, 1998). FTP and web sessions are the non real-time services with BER of $10^{-6}$ and target $E b / N o$ of $10 \mathrm{~dB}$. The activity factor of voice is 0.5 and 1.0 for the other services. Other traffic simulation parameters are provided in Table 2. Models for FTP, NRTV and web browsing traffic can be found in (TSG-C.R1002, 2003); however, for simplicity, we modelled them as constant bit rate services. The minimum 
acceptable rate of FTP and web sessions is half their required average rate. The maximum $\mathrm{Eb} / \mathrm{No}$ degradation corresponds to $0.5 \mathrm{~dB}$.

\begin{tabular}{|c|c|c|c|c|}
\hline Parameter & Voice & NRTV & FTP & web \\
\hline Average bit rate (kb/s) & 13.3 & 64 & 115.2 & 57.6 \\
Call/Session duration (min) & 2 & 5 & 5 & 2 \\
\hline
\end{tabular}

Table 2. Call simulation parameters

We increased the average handoff rate to the central cell from 1 to $10 \mathrm{MS} / 10$ s by increasing the cell density from 25 to $400 \mathrm{MSs}$ / cell in indoor cells and from 100 to $1200 \mathrm{MSs}$ / cell in vehicular cells. The new call rate $\lambda$ is set to be equal to the handoff rate in each simulation run. At a handoff rate of 10, the central and surrounding cells are overloaded with traffic according to the indoor and outdoor market requirements in busy hours mentioned in (TSG-C.R1002, 2003). Such high loads present a worst case interference scenario, so the results shown at those handoff rates can be considered as a low bound of wideband CDMA performance.

The overhead on the forward link due to the percentage of power dedicated to the pilot, sync and paging channels is assumed to be $17 \%$ and the maximum transmit power limit of a mobile is set to $24 \mathrm{dBm}$ (TR45.5, 1998). The background thermal noise, used in checking the limit of user equipment transmit power, is $-100 \mathrm{~dB}$ (Padovani, 1994). The maximum base station transmit power is assumed to be $20 \mathrm{~W}$. The spreading bandwidth $W$ is $3750 \mathrm{~kb} / \mathrm{s}$ corresponding to a third-generation 3xRTT cdma2000 technology. The orthogonality factor is taken to be 0.8 (Yang \& Lee, 1997). All results shown are the average of 20 simulation runs conforming to a confidence level of $95 \%$ with errors varying from 0.012 to 0.05 , each having a simulation time of 1 hour.

\subsection{Admission control performance}

We begin by testing our admission control module without the use of the Degradation module. Two aspects are analyzed: the admission condition threshold Th and the new-call/ handoff admission policy. They are analyzed with respect to blocking, dropping and outage probabilities. The blocking probability is the probability to deny the access to a new call while the handoff drop probability concerns the possibility of not being able to satisfy the requirements of a new incoming handoff call; both are due to capacity limits. The rejected handoff call continues with its already connected legs; though causing more interference, it becomes soon in outage. As mentioned before, the blocking probability on the uplink covers new call blocking when either the required transmitted power exceeds the maximum transmit power limit or the uplink admission condition of noise rise is not satisfied. The range of uplink $\eta_{K}$ and downlink $\eta$, obtained from simulations, is 0.16 to 0.58 and 0.21 to 0.73 respectively. In order to analyze the effect of Th on both the uplink and downlink capacity, the two probabilities have been measured for three threshold values, 0.6, 0.75 and 0.9 , as shown in Fig.4 and Fig.5. As the threshold increases, more services are admitted on both the uplink and downlink. This results in lower block and drop probabilities, however, with the cost of increasing mobile outage. Note that the mobile transmit power limit counts for $9-13 \%$ of the blocking probability of new calls and for $4-6.5 \%$ of the handoff drop probability on the uplink. It is worth noting that the outage rate increases drastically with a threshold of 0.9 , especially, at high loads. It reaches $8 \%$ and $6 \%$ on uplink and downlink respectively. This is because the coverage area, over which the minimum acceptable Eb/No is obtained, reduces with increasing number of services. At low threshold, 0.6, the outage can be neglected, however, the block and drop probabilities increase significantly at medium and high loads. The 0.75 


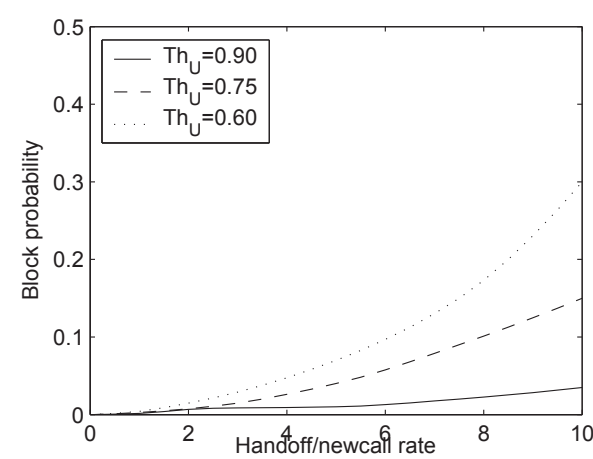

(a)

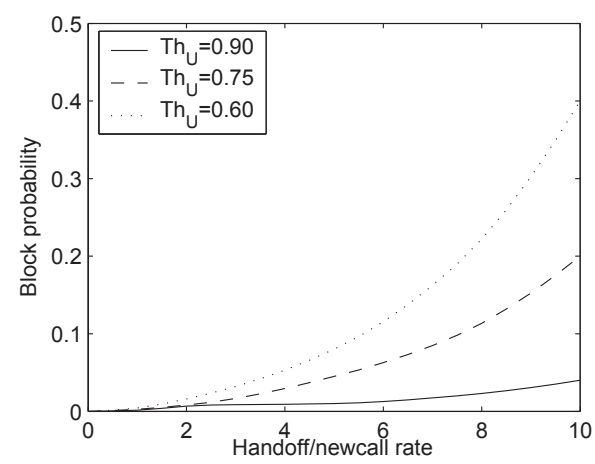

(c)

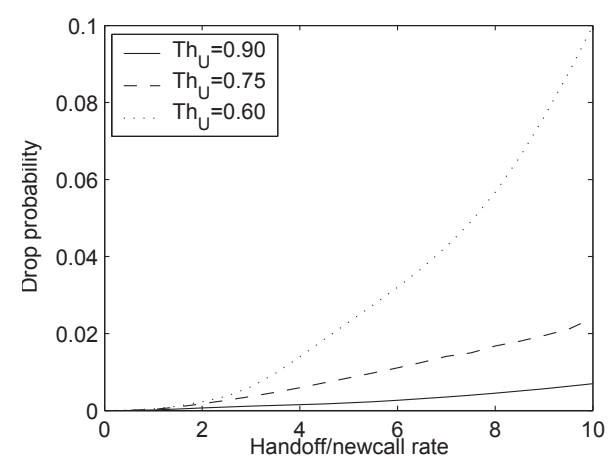

(b)

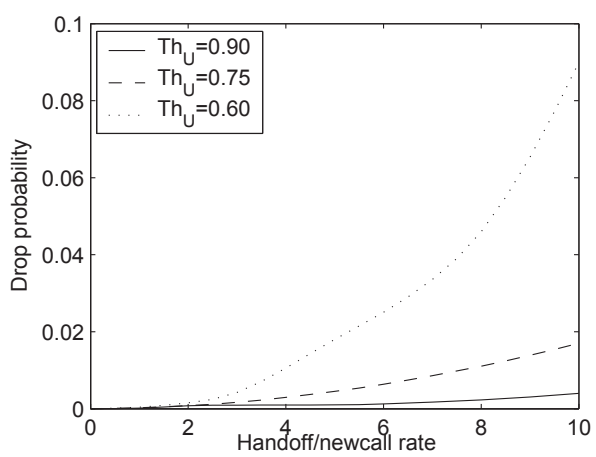

(d)

Fig. 4. Block and handoff drop probabilities on the uplink when varying the new/handoff rate (MS/10s) using different load thresholds in indoor $(a, b)$ and outdoor $(c, d)$ environments.

threshold gives a good coverage-capacity compromise. Moreover, with a threshold of 0.75 , the outage rate at high loads is below $1 \%$ on both uplink and downlink, which is better than the $95 \%$ coverage required by ITU. Thus, the remaining results are obtained with a 0.75 threshold and the outage rate is not investigated further since it remains below $1 \%$ with this threshold value.

In what concerns the new-call/handoff admission policy, it can be seen in Fig.4 and Fig.5, that the proposed policy, which gives incoming handoff calls a priority over new calls, results in achieving handoff drop probabilities much lower than new-call blocking ones on both uplink and downlink. The handoff drop probability does not exceed $1 \%$ in medium loads and is around $2 \%$ in very high loads. Nevertheless, in order to assess it with respect to other possible policies, we compare the handoff drop and new-call block probabilities when deploying the same proposed admission conditions (for CDMA) on the same simulated environments, but with different policies. Two other policies have been tested: the guard channel (GC) approach and the equal priority (EP) scheme. With a GC policy, a certain cell capacity is reserved solely for incoming handoff calls and the left capacity is for common use for all calls. That is, the load threshold is further decreased by a guard factor for new calls. This strategy was suggested by (Cheng \& Zhuang, 2002; Kulavaratharasah \& Aghvami, 1999). In contrast, with the EP 


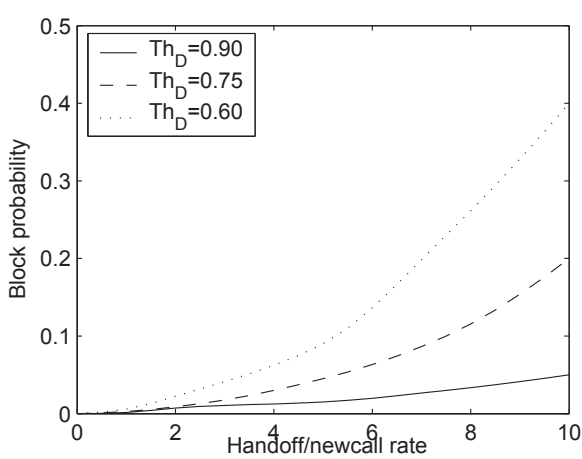

(a)

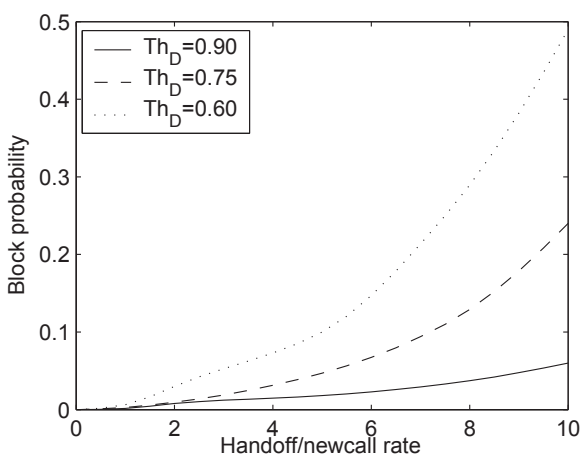

(c)

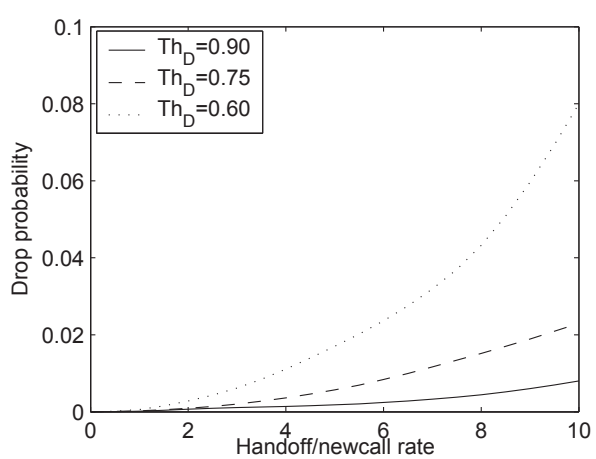

(b)

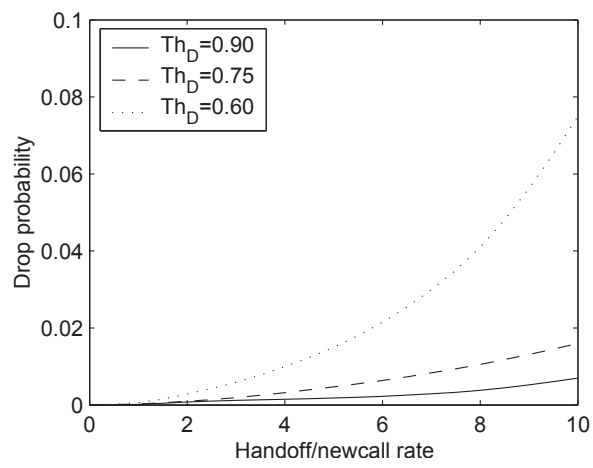

(d)

Fig. 5. Block and handoff drop probabilities on the downlink when varying the new/handoff rate (MS/10s) using different load thresholds in indoor $(a, b)$ and outdoor $(c, d)$ environments.

policy, both handoff and new calls are accepted if enough capacity exists to accomodate thier needs, no portion of the capacity is restricted for access of either type of call. This approach was selected by (Chang \& Chen, 2006; Das et al., 2000). Our proposed policy gives incoming handoff calls a priority over new calls when call rejection becomes necessary, that is, when no capacity is available.

Note that for simplicity, from here after, the drop and block probabilities include both the uplink and downlink ones. Fig.6 shows that, in indoor environment, the handoff drop probability of our policy is below that of EP scheme by a difference that varies from $1 \%$ for a handoff rate of 1 to about $20 \%$ for a rate of 10 . This is because our module gives the priority to handoff services compared to the EP scheme which does not differentiate handoff and new services. However, our block probability is higher than that of EP scheme by a difference that varies from $1 \%$ to $5 \%$. It is clear that our gain in handoff admission surpasses the loss in new service admission.

Fig. 6 demonstrates also the drop/block probability for 3 guard capacities of GC scheme. We observe that our handoff admission probability has a comparable performance with the GC scheme. It outperforms that of the 0.2 and 0.4 guard capacities by up to $7 \%$ and $2.5 \%$ respectively. However, the 0.6 guard capacity surpasses it by up to $2 \%$ for a handoff rate 


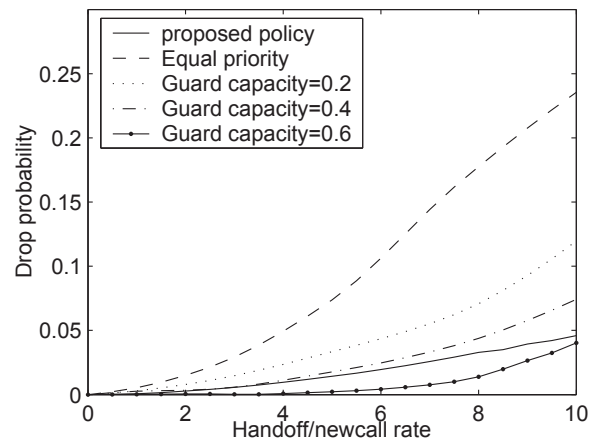

(a)

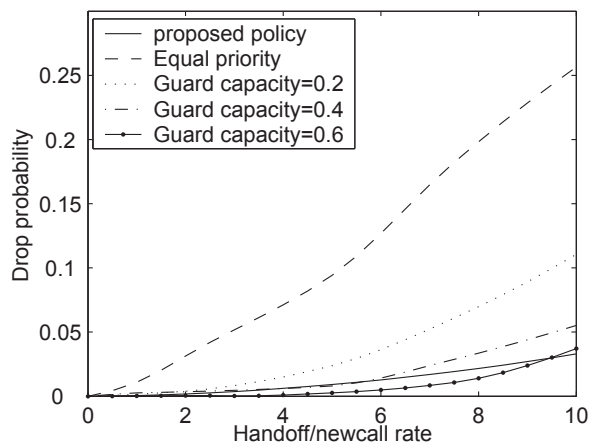

(c)

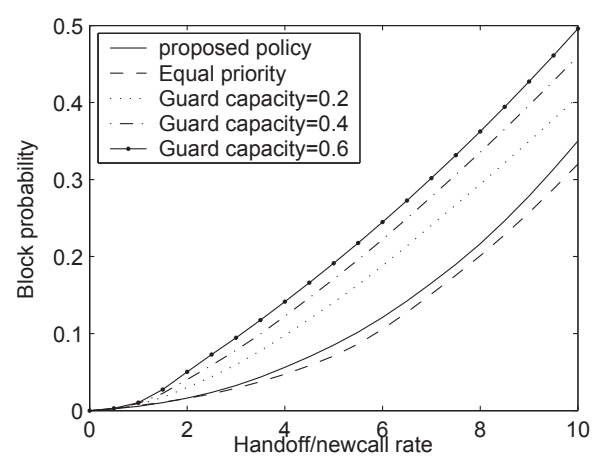

(b)

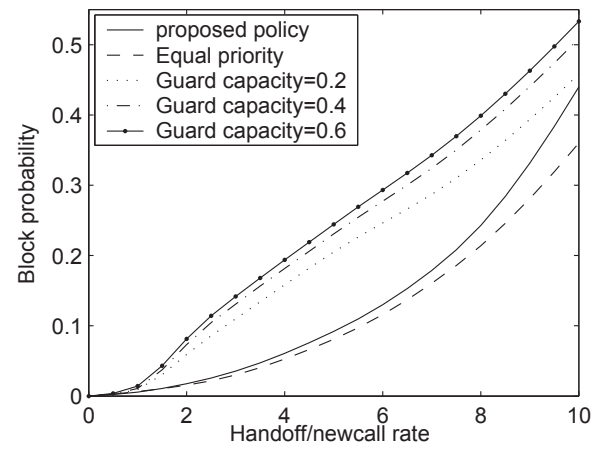

(d)

Fig. 6. Drop and block probability when varying the new/handoff rate (MS/10s) using different policies in indoor $(a, b)$ and outdoor $(c, d)$ environments.

that varies from 2 to 8 . This difference drops to $0.5 \%$ in indoors and vanishes in outdoors at high handoff rates. As for the block probability of new services, it can be seen that our scheme outperforms all the guard capacities by up to $15 \%$ indoors and $18 \%$ outdoors for new call rates varying from 2 to 8 . This is because, with a small handoff rate, the GC scheme results not only in high blocking of new services but also in low resource utilization because handoff services are allowed to use the reserved capacity exclusively. On the other hand, with a big number of handoff MSs that exceed the guard capacity, this scheme looses its advantage because it becomes difficult to guarantee the requirements of handoff users. The same observations can be noticed in outdoor environments. However, the drop probability of our approach is marginally better at high handoff rates with a difference of $1.4 \%$. This is due to the fact that the outdoor cell is less dense than the indoor cell when using our motion model, which gives the AC module a little more capacity for admitting more handoff services.

We have combined both the block and drop probabilities in order to measure the total number of admitted services. Fig.7 shows that the proposed policy outperforms both the GC scheme and the EP approach in terms of total number of accepted services in the cell, either handoff or new ones, especially in high loads. It surpasses the EP approach by $14.2 \%$ and $13 \%$ in indoor and outdoor environments respectively. It outperforms the GC scheme by up to $12 \%$ and $15 \%$ 


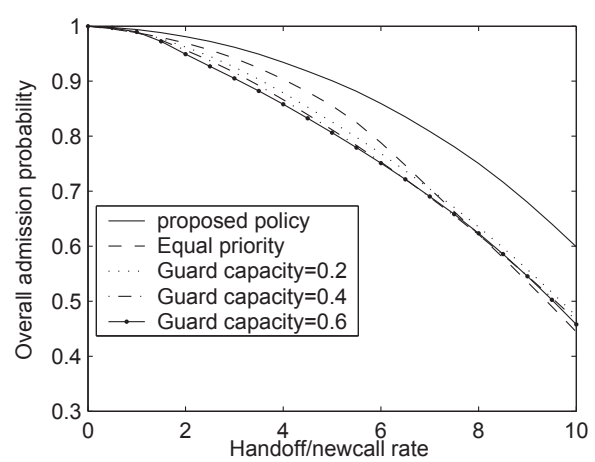

(a)

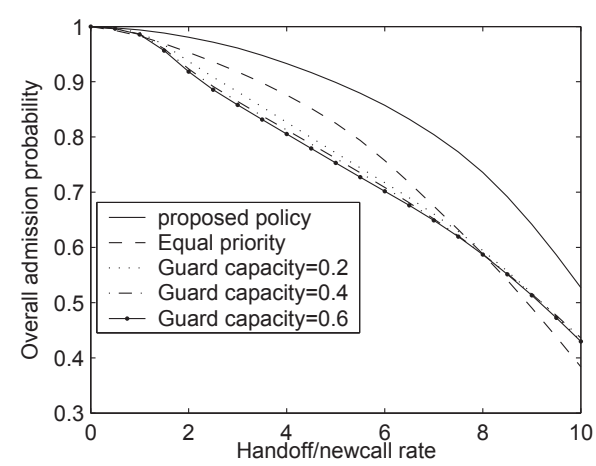

(b)

Fig. 7. Admission probability when varying the new/handoff rate (MS/10s) using different admission schemes in indoor (a) and outdoor (b) environments.

for 4-8 new / handoff rates. As also shown in Fig.7 it is clear that, at higher rates, this difference does not increase, where no capacity to be managed is left.

Recall that the time complexity of our AC module is $O(M)$ where $M$ is the cell density. So, in the worst case where the cell density is 400 and $1200 \mathrm{MSs} /$ cell in indoor and outdoor environments, the computation load is $O(1)$ in both environments. This is valid for forward services and reverse services that are not in soft handoff with other cells. However, for reverse connections that have, for instance, 2 soft handoff legs as in our simulations, this computing load would be multiplied by the number of handoff legs, which proves that soft handoff is computationally expensive as mentioned in (Kumar \& Nanda, 1999).

\subsection{Performance of D/l modules}

Next, we evaluate the effect of deploying our D/I modules on the handoff/new admission probability resulting from our admission control scheme. First, we study the effect of varying Rsafe on the overall drop+block probability, then, for simplicity, two values are selected for Rsafe in order to study in details the benefits on admission probability as well as on cell throughput. Fig. 8 shows the drop+block probability at a 7 new/handoff rate in indoor environment. Note that similar results were found in outdoors as well. When Rsafe $=\mathrm{Rc}$, this corresponds to no degradation, while Rsafe $=0$ means that all MSs inside the cell are subject to degradation with no preference. It can be seen that as Rsafe decreases, the drop+block probability is reduced significantly. This is because as Rsafe decreases, zone 1 becomes larger and, hence, the probability of locating MSs that can be degraded rises, giving more possibility to acquire capacity for new and handoff calls. However, below $0.3 R \mathrm{c}$, the benefit of further decreasing of Rsafe on drop+block probability diminishes because the remaining safe area (zone 2) has become much smaller than zone 1. In what follows, we present results for Rsafe equal to $0.75 \mathrm{Rc}$ and $0.5 \mathrm{Rc}$, which correspond to a safe zone of about half and quarter of the cell area respectively.

At low loads, the D/I scheme has a negligible effect on the admission performance. However, its contribution is manifest at high loads. Fig.9 shows that, when Rsafe is $0.5 R c$, the drop probability is less than that shown in Fig.6 with 3.5\% in indoor environment and $2.4 \%$ in outdoor environment. Moreover, it can be seen that, with the deployement of the Degradation module, the handoff admission probability surpasses the ones using guard capacities. This is 


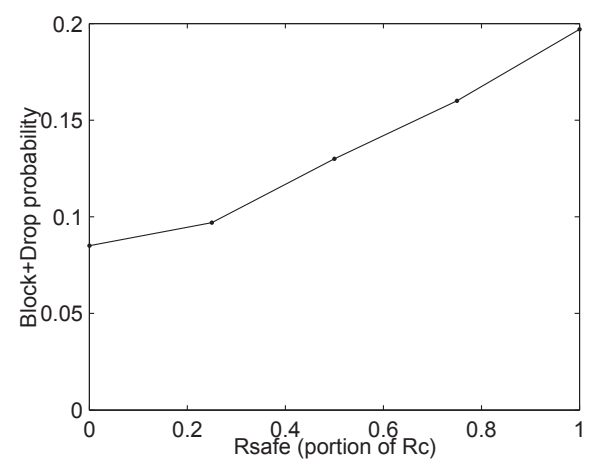

Fig. 8. Effect of varying Rsafe on the overall drop+block probability at a new/handoff rate of $7 \mathrm{MSs} / 10 \mathrm{~s}$ in indoor environments.

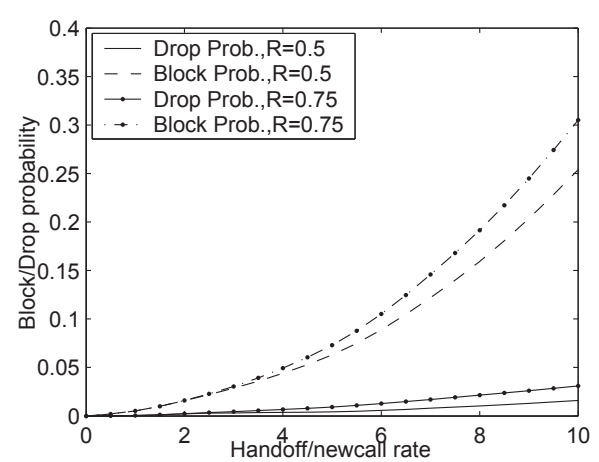

(a)

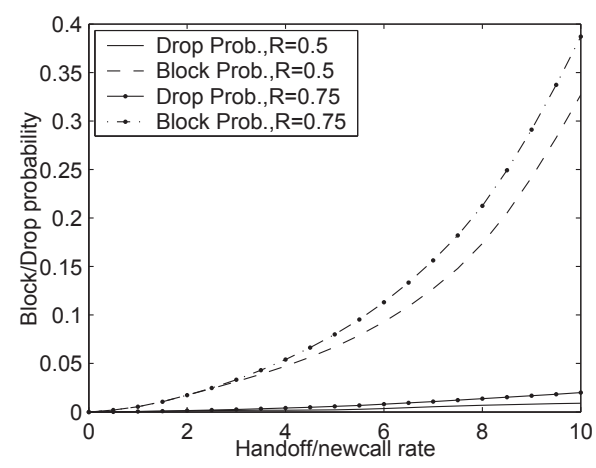

(b)

Fig. 9. Adaptation effect on drop and block probability when varying the new/handoff rate (MS/10s) in indoor (a) and outdoor (b) environments for Rsafe $=0.5 \mathrm{Rc}$ and $0.75 \mathrm{Rc}$.

because, with our design, there is no reservation of capacity for handoff services; instead, the call drop probability is decreased by degrading the QoS levels of services located near cell boundary, which reduces interference as well. As for new services, their block probability shows a significant improvement when compared to that shown in Fig.6; it has been reduced by a further $9.6 \%$ in indoor environments and $11.3 \%$ in outdoor environments. Furthermore, it can be seen that the new service admission probability is comparable to that of EP scheme shown in Fig.6 with the deployment of the Degradation module at Rsafe $=0.75 \mathrm{Rc}$ and even better at 0.5 Rc. Note that the observed outage when deploying D/I was always below $1 \%$.

When Rsafe is set to $0.75 \mathrm{Rc}$, the number of candidates for degradation decreases, which reduces the capacity that could be acquired for admitting new/handoff services. An improvement can still be observed in Fig.9. However, it is by far less than that of 0.5Rc. Fig.10 also shows the percentage of degraded MSs for both values of Rsafe. It can be seen that this percentage, in outdoor environments, goes up to $15.6 \%$ and $10 \%$ of total number of MSs for Rsafe of $0.5 \mathrm{Rc}$ and $0.75 \mathrm{Rc}$ respectively. This percentage drops to $7.5 \%$ and to $5.2 \%$, in 


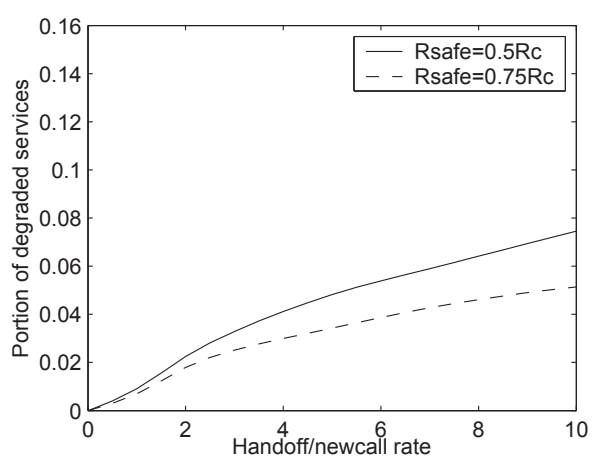

(a)

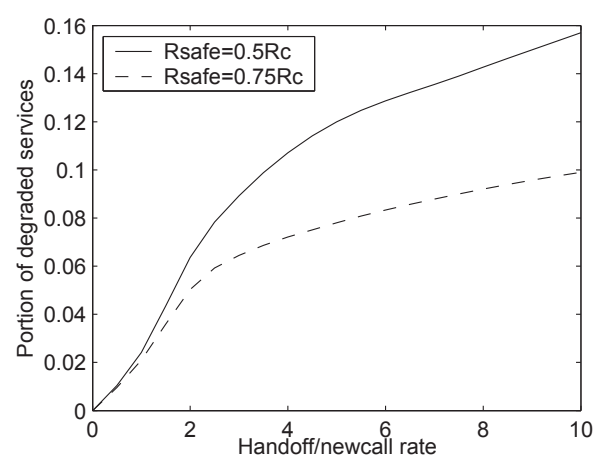

(b)

Fig. 10. Portion of degraded services in indoor (a) and outdoor (b) environments for Rsafe $=0.5 R c$ and $0.75 R c$ when varying the new $/$ handoff rate (MSs/10s).

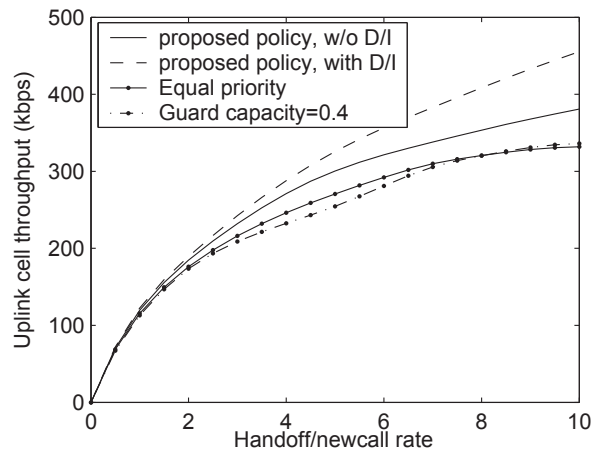

(a)

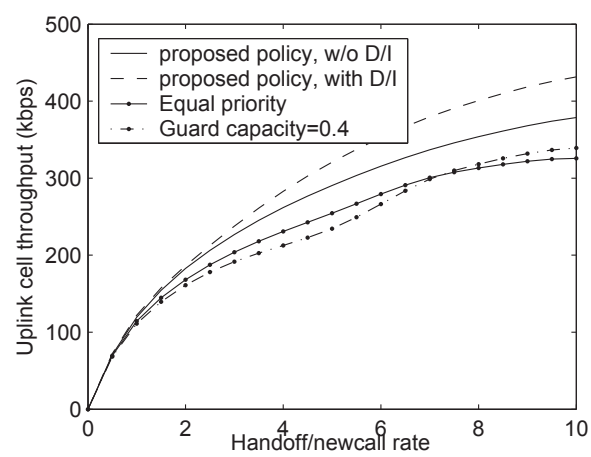

(c)

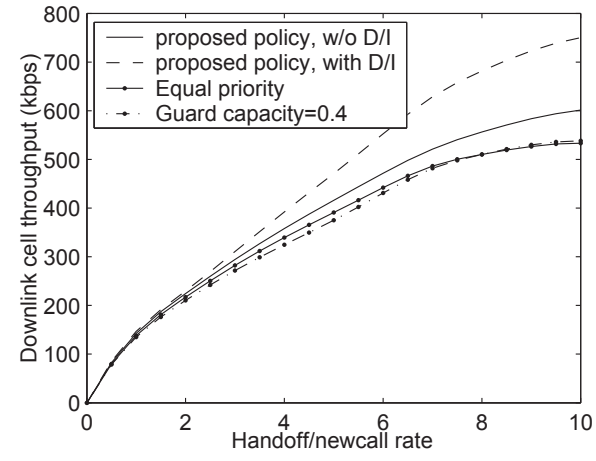

(b)

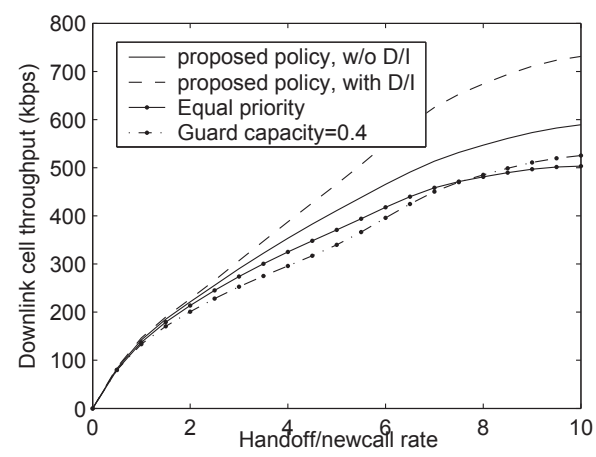

(d)

Fig. 11. Cell throughput on uplink and downlink when varying the new/handoff rate (MSs /10s) in indoor $(\mathrm{a}, \mathrm{b})$ and outdoor $(\mathrm{c}, \mathrm{d})$ environments. 
indoor environments, for Rsafe of $0.5 \mathrm{Rc}$ and $0.75 \mathrm{Rc}$ respectively at high loads. This explains the difference in the observed improvement for both kinds of environments.

Note that, as the handoff rate increases, the proportion of degraded services increases till a point where the cell begins to be highly loaded. At this point, the AC module starts to decrease the rate of admitted minimum throughput services. Moreover, the Eb/No degradation of the near-real-time services is limited to $0.5 \mathrm{~dB}$ only, and degradation is only allowed if their measured signal to interference ratio is not already degraded. This limits the possibility of degradation for services since they are not degraded below their minimum acceptable requirements. Thus, in highly loaded situations, the proportion of degraded services increases as well but with a rate lower than that of lighter load situations. This also demonstrates that our design succeeds in limiting the number of degraded MSs and, hence, reducing the required signalling messages which saves time and capacity.

In order to verify the effect of D/I deployment on cell throughput, the throughput of the services inside the cell was measured, for Rsafe $=0.5 \mathrm{Rc}$, and compared to the throughput of the admission control scheme without D/I. It was also compared to the throughput of EP policy and GC scheme having a guard capacity equal to 0.4 . The cell throughput only includes the bit rate of the calls that stay in the cell till termination or ongoing to another cell without being in outage. It represents the average of the instantaneous aggregated bit rate of only the calls currently served by the base station. Fig.11 shows the throughput on uplink and downlink in indoor and outdoor environments when varying the new/handoff rate. At moderate loads, the D/I can only enhance by around $20-30 \mathrm{~kb} / \mathrm{s}$ each of the uplink and downlink throughputs. Nevertheless, in high loads, this enhancement is boosted up to $62 \mathrm{~kb} / \mathrm{s}$ and $151 \mathrm{~kb} / \mathrm{s}$ in indoors, and $51 \mathrm{~kb} / \mathrm{s}$ and $142 \mathrm{~kb} / \mathrm{s}$ in outdoors, on the uplink and downlink respectively. That is, an improvement of more than $210 \mathrm{~kb} / \mathrm{s}$ in the total cell throughput can be obtained in high loads. As also shown in Fig.11, the throughput of the proposed policy, with D/I, clearly outperforms those of EP and GC approaches. This demonstrates that the $\mathrm{D} / \mathrm{I}$ deployment can rise the cell throughput as well as increasing the admission probability as seen above. However, this is achieved at the expense of unfairness between services, since degrading or improving the service level is not done uniformally across services, it depends on the MS location with respect to the safe area with aim of reducing interference.

The computation load of the Improvement module is the same as the one of the AC module without the soft handoff factor. However, the Degradation module has higher computation load of $O(N \log N)$ where $N$ is the cell density. So, in the worst case where the cell density is 400 and $1200 \mathrm{MSs} /$ cell in indoor and outdoor environments respectively, the computation load is $O(1)$ for forward and reverse services.

Another factor in evaluating the performance of the D/I modules is the response time for QoS adaptation. Since such QoS adjustment requires at most one signalling message per service, the time taken for a service to respond to such change is the time to send the control message to the MS of the service and processing it.

\section{Conclusion and future work}

In this chapter, we presented the design and evaluation of a service management scheme that is responsible for controlling the admission of new and handoff services and for service adaptation. The results show that our admission control proposal outperforms both the GC scheme and the EP approach in terms of total number of accepted services in the cell, either handoff or new ones, especially in high loads. It surpasses the EP approach by $14.2 \%$ and $13 \%$ and outperforms the GC scheme by $12 \%$ and $15 \%$ in indoor and outdoor environments 
respectively. Moreover, while limiting interference, signalling and computation overhead, the $\mathrm{D} / \mathrm{I}$ modules succeeded in further improving the admission probability. The drop probability is lower than that when deploying the AC module only with 3.5\% in indoor environment and $2.4 \%$ in outdoor environments. As for new services, their block probability shows a significant improvement, it is reduced by a further $9.6 \%$ in indoor environments and $11.3 \%$ in outdoor environments. The overall admission rate enhancement is achieved with low cost in terms of computition time and signalling messages, however, at the expense of unfairness among services.

In the research presented in this chapter, we did not consider automatic repeat request (ARQ) for retransmission on the radio link and forward error correction (FEC) techniques. These error correction mechanisms will be considered in a future work, since they can further enhance system capacity by decreasing target signal to noise ratios. Another research direction is to further examine new procedures for service admission on multiple cells level. This requires access coordination between BSs including sharing load information among neighbour cells, so that light loading in neighboring cells can be exploited to favor lower drop and block probabilities for handoff and new services respectively while still meeting interference constraints.

\section{References}

Aissa, S., Kori, J. \& Mermelstein, P. (2004). Call admission on the uplink and downlink system based on total received and transmitted powers, IEEE Transactions on Wireless Communications Vol. 3(No. 6): 2407-2416.

Chang, J. \& Chen, H. (2006). A borrowing-based call admission control policy for mobile multimedia wireless networks, IEICE Transactions on Communications Vol. E89-B(No. 10): 2722-2732.

Cheng, Y. \& Zhuang, W. (2002). Diffserv resource allocation for fast handoff in wireless mobile internet, IEEE Communications Magazine Vol. 4(No. 5): 130-136.

Comaniciu, C., Mandayam, N. B., Famolari, D. \& Agrawal, P. (2000). Qos guarantees for third generation $(3 \mathrm{~g})$ cdma systems via admission and flow control, Proceedings of VTC, IEEE, Boston, MA, pp. 249-256.

C.S0005-Ev2.0 (2010). Upper layer (layer 3) signaling standard for cdma2000 spread spectrum systems, 3GPP2 Specifications .

Das, S., Jayaram, R., Kakani, N. \& Sen, S. (2000). A call admission and control scheme for qos provisioning in next generation wireless networks, Journal of wireless Networks Vol. 6(No. 1): 17-30.

Gilhousen, K., Jacobs, I., Padovani, R., Viterbi, A., Weaver, J. \& Wheatley, C. (1991). On the capacity of a cellular cdma system, IEEE Transactions on Vehicular Technology Vol. 4: 303-312.

Him, K. \& Koo, I. (2005). CDMA Systems Capacity Engineering, ARTECH HOUSE, INC.

Homnan, B., Kunsriruksakul, V. \& Benjapolakul, W. (2000). The evaluation of soft handoff performance between is-95a and is-95b/cdma2000, Proceedings of International Conference on Signal Processing and Communications, IASTED, Marabella, Spain, pp. 38-42.

Issa, O. \& Gregoire, J. (2006). A service admission scheme for cellular networks, Proceedings of CCECE, IEEE, Ottawa, Canada, pp. 750-753.

Kastro, Y., Isiklar, G. \& Bener, A. (2010). Resource allocation in cellular networks based on marketing preferences, Journal of wireless Networks Vol. 16(No. 1): 27-38. 
Kelif, J. \& Coupechoux, M. (2009). On the impact of mobility on outage probability in cellular networks, Proceedings of Wireless Communications and Networking Conference, IEEE, Budapest, Hungary, pp. 1308-1313.

Kim, D., Hossain, E. \& Bahragavan, V. (2003). Dynamic rate adaptation based on multidimentional multicode ds-cdma in cellular wireless networks, IEEE Transactions on Communications Vol. 51(No. 2): 247-260.

Kulavaratharasah, M. \& Aghvami, A. (1999). Teletraffic performance evaluation of microcellular personal communication networks (pcn's) with prioritized handoff procedures, IEEE Transactions on Vehicular Technology Vol. 48(No. 1): 137-152.

Kumar, S. \& Nanda, S. (1999). High data-rate packet communications for cellular networks using cdma: algorithms and performance, IEEE Journal of Selelected Areas in Communnications Vol. 17(No. 3): 472-492.

Kwon, T., Choi, Y., Bisdikian, C. \& Naghshineh, M. (2003). Qos provisioning in wireless/mobile multimedia networks using an adaptive framework, Journal of wireless Networks Vol. 9(No. 1): 51-59.

Lee, J., Jung, T., Yoon, S., Youm, S. \& Kang, C. (2000). An adaptive resource allocation mechanism including fast and reliable handoff in ip-based $3 \mathrm{~g}$ wireless networks, Proceedings of 3Gwireless, IEEE, Silicon Valley, pp. 306-312.

Nasser, N. \& Hassanein, H. (2004). Combined admission control algorithm and bandwidth adaptation algorithm in multimedia cellular networks for qos provisioning, Proceedings of CCECE, IEEE, Niagara Falls, Canada, pp. 1183-1186.

Nasser, N. \& Hassanein, H. (2006). Robust dynamic call admission control framework for prioritized multimedia traffic in wireless cellular networks, International Journal of High Performance Computing and Networking (IJHPCN) Vol. 4(No. 1/2): 3-12.

Padovani, R. (1994). Reverse link performance of is-95 based cellular systems, IEEE Personal Communications Magazine Vol. 1: 28-34.

Sipila, K., Honkasalo, Z., j. Laiho-Steffens \& Wacker, A. (2000). Estimation of capacity and required transmission power of wcdma downlink based on a downlink pole equation, Proceedings of VTC, IEEE, Tokyo, Japan, pp. 1002-1005.

TR45.5 (1998). The cdma2000 itu-r rtt candidate submission, TIA .

TSG-C.R1002 (2003). 1xev-dv evaluation methodology (v14), 3GPP2 Specifications .

Yang, J. \& Lee, W. (1997). Design aspects and system evaluations of is-95 based cdma systems, IEEE Universal Personal Communications Record Vol. 6: 381-385. 


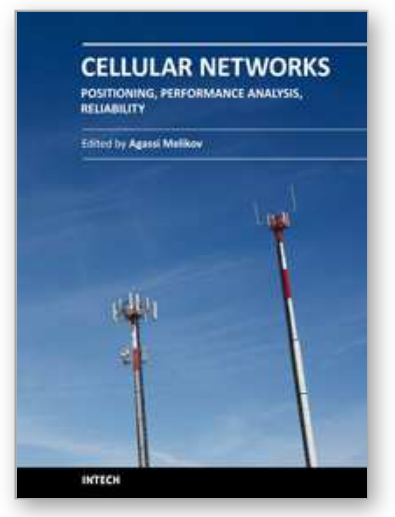

\author{
Cellular Networks - Positioning, Performance Analysis, Reliability \\ Edited by Dr. Agassi Melikov
}

ISBN 978-953-307-246-3

Hard cover, 404 pages

Publisher InTech

Published online 26, April, 2011

Published in print edition April, 2011

Wireless cellular networks are an integral part of modern telecommunication systems. Today it is hard to imagine our life without the use of such networks. Nevertheless, the development, implementation and operation of these networks require engineers and scientists to address a number of interrelated problems. Among them are the problem of choosing the proper geometric shape and dimensions of cells based on geographical location, finding the optimal location of cell base station, selection the scheme dividing the total net bandwidth between its cells, organization of the handover of a call between cells, information security and network reliability, and many others. The book focuses on three types of problems from the above list Positioning, Performance Analysis and Reliability. It contains three sections. The Section 1 is devoted to problems of Positioning and contains five chapters. The Section 2 contains eight Chapters which are devoted to quality of service (QoS) metrics analysis of wireless cellular networks. The Section 3 contains two Chapters and deal with reliability issues of wireless cellular networks. The book will be useful to researches in academia and industry and also to post-gradute students in telecommunication specialitiies.

\title{
How to reference
}

In order to correctly reference this scholarly work, feel free to copy and paste the following:

Omneya Issa (2011). Mobility and QoS-Aware Service Management for Cellular Networks, Cellular Networks Positioning, Performance Analysis, Reliability, Dr. Agassi Melikov (Ed.), ISBN: 978-953-307-246-3, InTech, Available from: http://www.intechopen.com/books/cellular-networks-positioning-performance-analysisreliability/mobility-and-qos-aware-service-management-for-cellular-networks

\section{INTECH}

open science | open minds

\section{InTech Europe}

University Campus STeP Ri

Slavka Krautzeka 83/A

51000 Rijeka, Croatia

Phone: +385 (51) 770447

Fax: +385 (51) 686166

www.intechopen.com

\section{InTech China}

Unit 405, Office Block, Hotel Equatorial Shanghai

No.65, Yan An Road (West), Shanghai, 200040, China 中国上海市延安西路65号上海国际贵都大饭店办公楼 405 单元

Phone: +86-21-62489820

Fax: $+86-21-62489821$ 
(C) 2011 The Author(s). Licensee IntechOpen. This chapter is distributed under the terms of the Creative Commons Attribution-NonCommercialShareAlike-3.0 License, which permits use, distribution and reproduction for non-commercial purposes, provided the original is properly cited and derivative works building on this content are distributed under the same license. 